\title{
PREDICTION-BASED PROACTIVE CLUSTER TARGET TRACKING PROTOCOL FOR BINARY SENSOR NETWORK
}

\author{
Jing Teng, Hichem Snoussi and Cédric Richard \\ University of Technology of Troyes \\ ICD/LM2S \\ 12, rue Marie Curie, 10000, France \\ Email: jing.teng@utt.fr
}

\begin{abstract}
An efficient, economical and robust strategy for target tracking in binary sensor network is proposed in this paper. By adopting the binary variational filtering algorithm, considerable tracking quality is ensured, while decreasing communication between sensors compared to a particle filtering algorithm. Based on the proactive clustering, the entire sensor network is subdivided into several clusters. Only cluster heads are configured with more available energy and high processing capability, reducing thus the hardware expenditure. Furthermore, precise prediction of the target position and the cluster activation protocol ensure that the most potential cluster is activated to perform target tracking, reducing consumed energy during the hand-off operation. Employing of the binary variational filtering algorithm and the exception handle scheme ensure robustness in coping with the case of highly non-linear and non-Gaussian environments.
\end{abstract}

\section{INTRODUCTION}

Wireless sensor network (WSN) is an emerging technology, which has found its application in several potential areas. Among them, target tracking has attracted considerable attention in both literature and application domains. Aiming to detect the presence of an object and determine its path in an area of interest, target tracking thus requires effective coordination among sensor nodes. In order to retain a low configuration spending, the deployed sensors are equipped with small batteries. Therefore, a trade-off exists between the energy expenditure and the tracking quality. These constraints inspire ones to design a reasonably accurate estimation whilst minimizing the overhead of network configuration and maximizing the network lifetime by reducing the network communication.

In this paper, we investigate the problem of target tracking using a WSN composed of binary proximity sensors. As a power and bandwidth efficient solution, the binary proximity sensor can only make a binary decision based on the strength of sensed signal. Only when it senses the presence of the target that one bit is transmitted for further processing. The realistic binary sensor network (BSN) suffers from problems of a noisy link and low estimation precision. We have developed the binary variational filtering algorithm $(\mathrm{BVF})[1,2]$ that solves these problems while perfectly fits the highly nonlinear conditions and eliminates the error propagation.

In this contribution, in order to further reduce the energy consumption in BSN and minimize the configuration overhead, BVF is executed on a fully decentralized cluster scheme. At every instant, only one cluster is triggered according to the prediction of the target location and its tendency. The cluster head $(\mathrm{CH})$ collects information from its slave sensors and determines the target position, then predicts the target location of the next instant as well. Based on the reliable prediction, we can choose the most potential cluster to provide more accurate tracking while cutting down dramatically the number of hand-off operations between the CHs. Thus advanced resource conservation is achieved. The proposed architecture is also robust to the rare events of abrupt changes in target trajectory. In fact, in addition to the capability of BVF in dealing with non-linear/non-gaussian situations, the adopted exception handle scheme is robust to prediction failures.

The rest of the paper is organized as follows. In section 2 , we briefly summarize the related existing work. Then the BVF algorithm is introduced in section 3. In section 4, we describe the proposed prediction-based proactive cluster protocol (PPCP) and discuss the achieved resource conservation. The exception handle scheme introduced in section 5 is to ensure the system robustness. Finally, the proposed scheme is evaluated by simulations in section 6 . Section 7 concludes.

\section{RELATED WORK}

The problem of target tracking using binary sensors has been explored by many references $[3,4,5]$. Shrivastava et al. [3] explore the fundamental performance limits of target tracking by binary sensing models. The authors of [4] consider the piecewise linear path approximations and fix the scale of 
target velocity, their simulations depend on the error-free assumption as well. The sensing model employed in [5] is to provide direction information of the target of interest. However, without additional proximity information, it can not distinguish parallel trajectories.

Cluster-based tracking schemes have gained extensive attention recently, but to our knowledge, none of these algorithms aims at providing accurate target tracking and minimizing the energy consumed in the binary sensor networks. Heinzelman et al. [6] developed a low-energy adaptive clustering hierarchy $(\mathrm{LEACH})$. It randomly rotates the $\mathrm{CH}$ to evenly distribute the energy load among sensors. Tseng et al. [7] use the mobile agent technology. They select the agent by comparing the signal strength, and also dynamically choose two nearby sensors as slave sensors to cooperatively triangulate the target. But for the case of irregular network topologies, it may be energy or time intensive to choose the master and its slave sensors. The energy and memory spendings for the tracking histories in the moving agent is not calculated too. Chen et al. [8] provide a hierarchical WSN composed of a static backbone of $\mathrm{CHs}$ and dynamically formed clusters. Their design concentrates on dealing with the collision during the clustering phase. The performance of it degrades as the target speed increases.

Aiming at conserving the resources, we adopt binary sensors to form networks, and the entire BSN is subdivided into several clusters. Only $\mathrm{CH}$ are configured with more available energy and higher processing capability. Other slave sensors just perform the sensing function, and only if the target are detected, will they transmit one bit in a small distance to their master. Furthermore, by accurately predicting the target position and tendency, the energy consumed in hand-off operations reduces dramatically. To ensure accurate target tracking, the BVF algorithm is employed, which provides considerable precision while further decreasing communication between sensors compared to that of [9]. Robustness of the proposed approach is ensured by the exception handling scheme.

\section{BINARY VARIATIONAL FILTERING ALGORITHM}

The target position $x_{t}$ is assumed to be Gaussian distributed, with a random mean $\mu_{t}$ and a random precision matrix $\lambda_{t}$ to further capture the uncertainty about the state distribution:

$$
\left\{\begin{aligned}
x_{t} & \sim \mathcal{N}\left(x_{t} \mid \mu_{t}, \lambda_{t}\right) \\
\mu_{t} & \sim \mathcal{N}\left(\mu_{t} \mid \mu_{t-1}, \bar{\lambda}\right) \\
\lambda_{t} & \sim \mathcal{W}_{\bar{n}}\left(\lambda_{t} \mid \bar{S}\right)
\end{aligned}\right.
$$

Where $\bar{\lambda}$ is a fixed precision matrix to reflect the region of uncertainty for the new estimation around the old one. The state precision matrix is modeled by a Wishart distributed with $\bar{n}$ and $\bar{S}$ denoting respectively the degree of freedom and the precision matrix, both assumed to be fixed. Instead of the

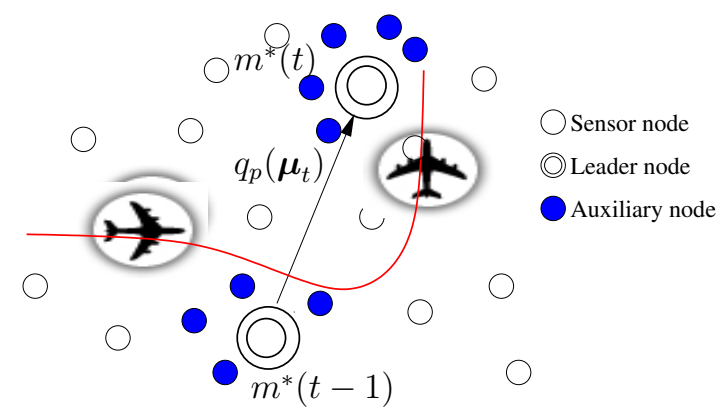

Fig. 1. Only one Gaussian statistics $q\left(\mu_{t-1}\right)$ is exchanged between leader cluster heads.

kinematic parameter models applied in [10], the use of a general state model [11] is more adaptive to the non-linear circumstance.

The observed binary data $z_{j, t}$ communicated to the leader $\mathrm{CH}$ from its slaves are distributed according to the following mode:

$$
\begin{aligned}
P\left(\left\{z_{j, t}\right\} \mid x_{t}\right) & =\prod_{j=1}^{m} \mathcal{N}\left(\beta_{j} s_{j, t}, \sigma_{\epsilon}^{2}\right), \\
s_{j, t} & = \begin{cases}1, & \text { if } y_{j, t}<\gamma \\
0, & \text { otherwise }\end{cases}
\end{aligned}
$$

where $\beta_{j}$ is the attenuation coefficient associated with the $j$ th sensor, $\sigma_{\epsilon}^{2}$ the noise variance, $y_{j, t}$ denotes the distance observed by the $j$-th sensor to the target at instant $t$.

Given the model above, the distribution of interest for tracking is the posterior $p\left(\alpha_{t} \mid \boldsymbol{z}_{1: t}\right)$, where $\alpha_{t}=\left(x_{t}, \mu_{t}, \lambda_{t}\right)$ denotes the extended hidden state and $\boldsymbol{z}_{t}=\left\{z_{j, t}\right\}$ means the observations gathered at $\mathrm{CH}$. It is approximated by a distribution $q(\alpha)$ that minimizes the Kullback-Leibler (KL) divergence error. Supposing that $q(\alpha)=\prod_{i} q\left(\alpha_{i}\right)$, where $\left\{\alpha_{i}\right\}$ denotes the subsets of $\alpha$ that are $x_{t}, \mu_{t}, \lambda_{t}$, then $q(\alpha)$ is of the form,

$$
q\left(\alpha_{i}\right) \propto \exp <\log p\left(\boldsymbol{z}_{1: t}, \alpha_{t}\right)>_{\prod_{j \neq i} q\left(\alpha_{j}\right)}
$$

where $<.>_{p}$ denotes the expectation operator relative to the distribution $p$.

Taking into account the separable approximate distribution at time $t-1$, the filtering distribution at time $t$ is written,

$$
\begin{aligned}
& \bar{p}\left(\alpha_{t} \mid \boldsymbol{z}_{1: t}\right) \propto p\left(\boldsymbol{z}_{t} \mid x_{t}\right) p\left(x_{t} \mid \mu_{t}, \lambda_{t}\right) p\left(\lambda_{t}\right) q_{p}\left(\mu_{t}\right), \\
& \text { with } q_{p}\left(\mu_{t}\right)=\int p\left(\mu_{t} \mid \mu_{t-1}\right) q\left(\mu_{t-1}\right) d \mu_{t-1} .
\end{aligned}
$$

The temporal dependence on the past is hence reduced to the incorporation of only one component approximation $q\left(\mu_{t-1}\right)$, which is Gaussian, limiting the communication between two successive leaders to simply sending the mean and the covariance of $q\left(\mu_{t-1}\right)$ (as shown in Fig.1). The update of the filtering distribution and its approximation are jointly performed, yielding thus a natural and adaptive compression of the filtering distribution [1], which is propagated in the BSN without lossy compression. The whole process is demonstrated in 


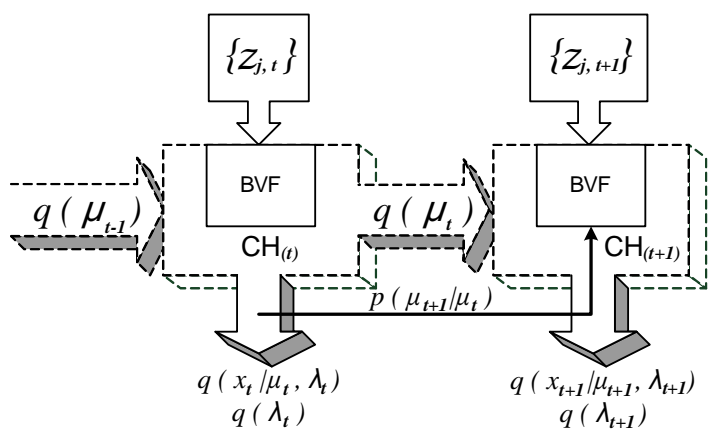

Fig. 2. BVF process during consecutive sampling instants.

Fig.2, while dashed lines mean that information transferring may not occur, as the leader stays the same during consecutive time slots. On contrast, the real line denotes the operation executed at every instant. By $p\left(\mu_{t+1} \mid \mu_{t}\right)$, the leader of next instant is chosen.

\section{PREDICTION-BASED PROACTIVE CLUSTER PROTOCOL}

A cluster scheme is employed here to facilitate collaborative signal processing and to ensure resource conservation in target tracking sensor network. The clustering protocol employed is proactive, which means that clusters are formed in advance. So instead of assuming the identical configuration for all sensors, we present a hierarchical BSN: a large number of slave sensors are randomly and densely deployed over the span of the network, and are subdivided into clusters according to their distances to $\mathrm{CHs}$; $\mathrm{CHs}$ with high capability are sparsely placed and will be triggered by certain events. There are several benefits of using this cluster scheme: firstly, the spending of hardware configuration drops sharply. For proactive clustering scheme, as there is no need for $\mathrm{CH}$ competition, only $\mathrm{CHs}$ are configured with more available energy and higher computation capability, slave sensors just sense and transfer data upon their CH's request. Secondly, bandwidth needed and energy consumed during communication phases reduced dramatically. Since signal processing task is distributed among CHs, only when hand-off operations occur do $\mathrm{CHs}$ need to change more information for a considerable long distance. The number of hand-off operations is reduced by the reliable prediction of the target tendency too. For most of the time, just the slave sensors that belong to the leader and have detected the target are required to transmit one bit in a small distance. Thirdly, the lifetime of BSN is extended to a great degree. Though slave sensors are densely spread out, at every sampling instant, only a few of them are requested to sense and only one $\mathrm{CH}$ is activated to locate the target. It is well known that when sensors are in the sleeping mode, they consume only $0.1 \%$ of the energy consumed in the active mode. Experimental data shows that sensors can last for more than a year on a $1 \%$ duty cycle [12].

\subsection{Coverage problem}

We assume that slave sensors are uniformly and randomly distributed with density $d$. A straightforward way to locate target by using binary sensors is to obtain a bounded intersection area from sensor readings. Hence the coverage problem is of critical significance. There have been many research works focusing on the coverage problem. In this paper, we directly employ the analysis of [13], according to which, with the sensor density $d$, the target is within the distance of

$$
\tilde{D}=\frac{1}{\sqrt{d}}\left(\frac{1}{2}+4 \sqrt{\frac{1}{\pi}-\frac{1}{4}}\right)
$$

to the closest sensor at probability $\geq 99 \%$. For a $100 \times 100 \mathrm{~m}^{2}$ fields, if we randomly deploy 400 slave sensors, the sensing radius of them need to be at least 3.09 meters. While for a set of $n$ sensor readings, only $n-1$ out of the total $n(n-1) / 2$ intersection areas are independent. Therefore, in order to locate the target, at least four sensor are required to sense the target within their range [8]. To guarantee the four-coverage problem we need to either increase the slave sensors density or to extend the sensing range of each sensor. Since the slave sensors can simply make judgement of the presence or the absence of a target, extending the sensing range results in smaller resolution in target tracking. On the other hand, increasing the number of slave sensors brings on tremendous rise in hardware and energy expenditure, since also the number of $\mathrm{CHs}$ has to increase. Otherwise, $\mathrm{CHs}$ have to be equipped with multiplied memory, higher signal processing capability and much more available energy to support the computation and communication requirement. The BVF algorithm [1,2] helps balancing the trade-off between the tracking accuracy and the cost. By the probabilistic strategy, the stringent constraint of four-coverage could be relaxed. We prefer extending the sensing range rather than increasing the sensor density as the tracking quality is ensured by the BVF algorithm.

\subsection{Details of PPCP}

The proposed protocol is composed of three pivotal component mechanisms: initial proactive clustering, predictionbased $\mathrm{CH}$ leader selection, slave sensors activating and replying.

\subsubsection{Initial proactive clustering}

After the deployment of all sensors, each slave sensor transmits its position (assumed to be known) to the $\mathrm{CHs}$ within its transmission range (which is set to be twice of its sensing range according to the result of [8]). Owing to the greater sensing ranges of $\mathrm{CHs}$, the position information transmitted 


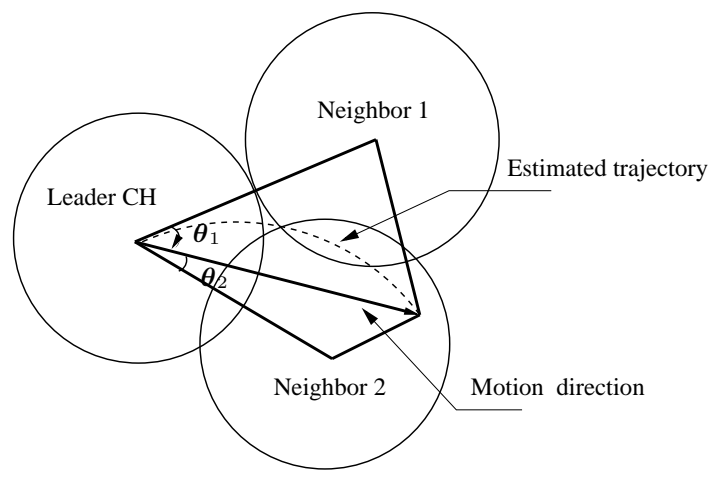

Fig. 3. Prediction-based CH leader selection.

by slave sensors is almost certainly to be received by at least one $\mathrm{CH}$. Based on distance and signal power comparing, each $\mathrm{CH}$ can form its cluster. During the clustering process, the $\mathrm{CHs}$ can also confirm its neighboring $\mathrm{CHs}$. When the clustering phase finishes, two kinds of lists are maintained in the CHs: the neighboring CHs list and the slave sensors list, which contain the node IDs and the node positions.

\subsubsection{Prediction-based CH leader selection}

To reach accurate target tracking while minimizing energy consumption, the $\mathrm{CH}$ selection strategy is of critical significance during the tracking phase. An intuitive solution is to choose the $\mathrm{CH}$ that is nearest to the target as the leader. But this strategy would incur unnecessary energy expenditure. First, at every sampling instant all the $\mathrm{CHs}$ need to measure the distance between the target and themselves then compare with each other to choose the nearest one. Thus the possibility of distributed signal processing is prevented and excessive energy is consumed. Second, frequent changes between $\mathrm{CHs}$ result in continuous communications of large information, which lead to unacceptable additional energy and bandwidth consumption.

To minimize energy expenditure, a hierarchical solution is proposed in our scheme. At the lower layer, the BVF algorithm $[1,2]$ is adopted, which means that instead of maintaining a large scale of particles and their weights in the particle filtering [14, 9], only one Gaussian statistic is transmitted to perform target tracking. At the higher layer, the leader $\mathrm{CH}$ is selected based on the exact prediction of target position and its trajectory tendency.

The leader $\mathrm{CH}$ not only processes the data from its slaves to estimate the target position, but also predicts the target future position to determine whether to execute the hand-off operation and which $\mathrm{CH}$ would take over. As mentioned above, the deployment of CHs ensures the coverage problem of target tracking. Generally, even if the target goes out of the sensing range of the leader $\mathrm{CH}$, there is a great probability that it is sensed by its neighbors. As demonstrated in Fig.3, based on the prediction of the target, we define a decision function that performs only between the leader and its neighboring $\mathrm{CHs}$ :

$$
\hat{S}(x)=\operatorname{argmax} \frac{\cos (\theta)}{d}
$$

where $\theta$ is the angle between the predicted target direction and the vector from the leader to its neighbor, $d$ is the distance from the predicted target to the neighbor. Based on this function, the most potential leader is chosen, as the target is most probably sensed by it for a considerably long period. This decision rule avoids unnecessary hand-off operations and conserves energy expenditure as well.

\subsubsection{Slave sensors activating and replying}

After the initial clustering phase, each slave sensor is informed by its master. Slave sensors rest in sleeping mode in general. They are awakened only when being scheduled to sense or when an activate message is received. For the former case, it senses the target presence in its vicinity while starting a local timer. When it has sensed the target, one bit is transmitted to the leader $\mathrm{CH}$; For the latter, by comparing the source of the message with its master ID, the slave sensor decides whether to change into sensing mode or not. If yes, when the target is detected, it transmits the sensing result to the leader automatically.

\section{EXCEPTION HANDLING SCHEME}

As described in [2], the BVF algorithm is more favorable than other algorithms in non-linear and non-gaussian environments. While by employing the cluster strategy, observation data come only from the activated cluster. There are cases that the chosen cluster can not provide reasonable tracking quality because of its restriction in observation scope. Especially in cases when the target has changed abruptly not only its velocity but also the moving direction. Exception handling scheme is introduced here to cope with these situations.

There are two kinds of exceptions: Firstly, when the newly chosen leader can not sense the target even by its slaves. Without observation data provided in the update phase, the BVF cannot yield out precise estimation. Then all $\mathrm{CHs}$ in the network are informed with a 'failure' signal, which means that an abrupt change has taken place during the target movement. The CHs who detected the target or whose slaves did, respond to the current leader with their observations. The current leader then chooses the best leader by performing the decision function mentioned above, where $d$ is the observation instead of the prediction. Furthermore, the 'failure' signal informs that the prediction provided by the BVF at the previous instant is not adaptable to the abrupt change. To ensure tracking accuracy, the mean of $q\left(\mu_{t-1}\right)$ is replaced by the position of the newly chosen $\mathrm{CH}$. Another exception occurs when the target moves between different clusters. In this case, the sensing 


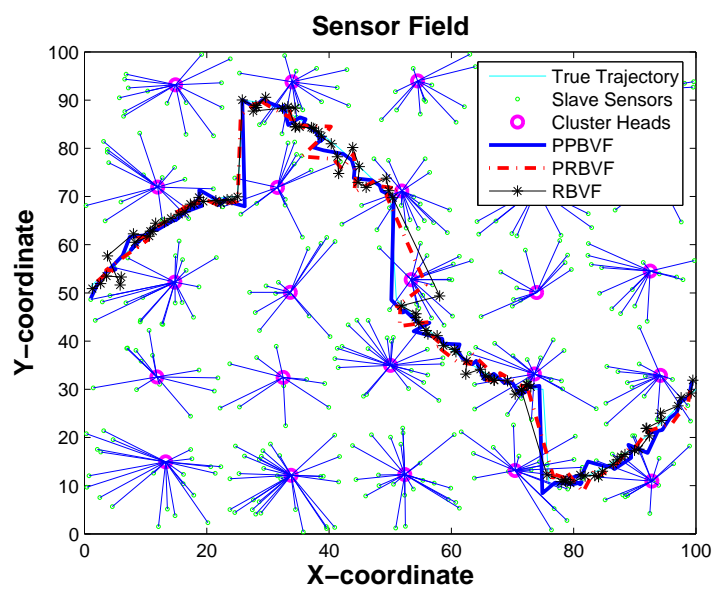

Fig. 4. Tracking trajectory of the three approach

nodes that have detected the target belong to different clusters. Thus, charging only one cluster with the task of data processing results in poor tracking quality. To handle this problem, the average of other cluster observations is transfered to the current leader and is considered as the mean of $q\left(\mu_{t-1}\right)$ to counteract the activated cluster observation. Unbiased estimation is reached then.

The reason for changing the mean of $q\left(\mu_{t-1}\right)$ is demonstrated in section 3. Since $q\left(\mu_{t-1}\right)$ is the sufficient statistic for computing the filtering distribution, it contains all temporal dependence information. Substituted by the observation data makes it robust to exceptions while integrating more information.

\section{EVALUATION AND SIMULATION}

To demonstrate the effectiveness and the energy consumption of the proposed protocol, we compared its performance with two similar approaches - Prediction-based Reactive BVF (PRBVF) and Reactive BVF (RBVF). By PRBVF, we mean that cluster is dynamically formed depending on the same decision function in section 4.2.2. RBVF means that the active cluster is formed by choosing the nearest $m$ sensors ( $m=10$ in the simulation here) to the target. Intuitively, besides the hardware expenditure, additional energy consumption during the clustering phrase is included in these two approaches. To calculate the energy expenditure during the whole process, we adopted two hypothesis[15]: 1) the communication between the active sensors is via single hop, 2) the energy consumed in scheduling and computing can be neglected relative to the energy consumed during communication.

According to the energy consumption model proposed in $[16,15]$, the energy consumed in transmission per bit is

$$
E_{T}=\epsilon_{e}+\epsilon_{a} d^{2}
$$

where $\epsilon_{a}$ is the energy dissipated in Joules per bit per $m^{2}, \epsilon_{e}$

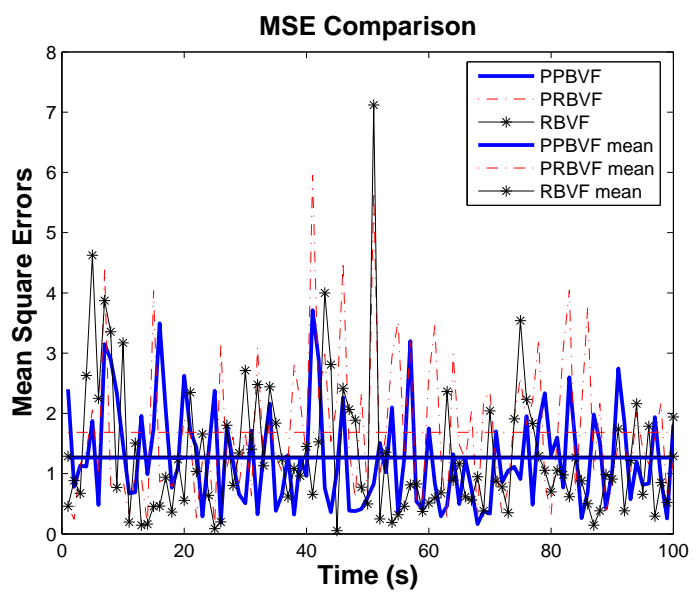

Fig. 5. Mean Square Error comparison

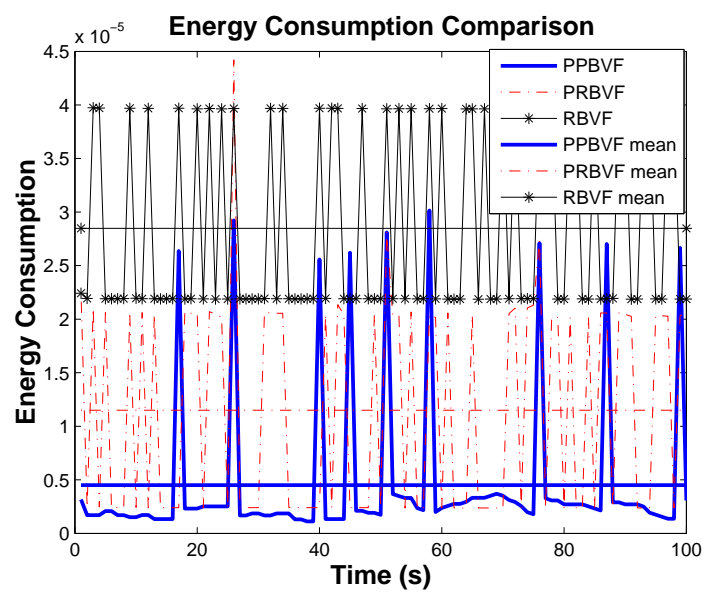

Fig. 6. Energy consumption comparison.

is energy consumed by the circuit per bit, $d$ is the transmission distance.

The power consumed when receiving data is given by

$$
E_{R}=\epsilon_{r} N
$$

where $\epsilon_{r}$ denotes the energy expended in receiving one bit of data.

Similarly, the power consumed in sensing is defined by

$$
E_{S}=\epsilon_{s} N
$$

where $\epsilon_{r}$ is the energy expending parameter for sensing one bit of data.

Following the energy model, we choose $\epsilon_{a}=$ $100 \mathrm{pJ} / \mathrm{bit} / \mathrm{m}^{2}, \epsilon_{e}=50 \mathrm{~nJ} / \mathrm{bit}, \epsilon_{r}=135 \mathrm{~nJ} / \mathrm{bit}, \epsilon_{s}=$ $50 n J / b i t$. The energy consumption of the three approaches is demonstrated in Fig. 6

Considering a target tracking duration of 100 time slots, 400 sensors are randomly deployed in a 2 dimensional field 
$\left(100 \times 100 \mathrm{~m}^{2}\right)$ and belong to 25 sparsely positioned $\mathrm{CHs}$, while PRBVF and RBVF have also employed the same configuration but without fixed CHs. Fig. 4, Fig. 5 and Fig. 6 show that our protocol successfully balances the trade-off between the tracking quality and the energy consumption even with several abrupt changes in the trajectory.

\section{CONCLUSION}

An efficient, economical and robust strategy for target tracking is proposed in the context of BSN. The effectiveness of tracking is ensured by the BVF algorithm and the reasonably chosen clusters to perform distributed signal processing. As for economical reasons, in the hardware layer, the deployment of binary sensors saves greatly energy and bandwidth; in the software layer, the BVF algorithm decreases the information exchanged between CHs to one Gaussian statistic; Proactive clustering reduces the hardware expenditure; Predictionbased selection of $\mathrm{CH}$ terminates unnecessary hand-off operation considerably, thus most of the communication is constrained within the vicinity of $\mathrm{CH}$ leaders. The robustness is ensured by exception handling scheme.

\section{REFERENCES}

[1] Hichem Snoussi and Cédric Richard, "Ensemble learning online filtering in wireless sensor networks," in IEEE ICCS International Conference on Communications Systems, 2006.

[2] Jing Teng, Hichem Snoussi, and Cédric Richard, "Binary variational filtering for target tracking in sensor networks," in IEEE Workshop on Statistical Signal Processing, 2007.

[3] N. Shrivastava, R. Mudumbai U. Madhow, and S. Suri, "Target tracking with binary proximity sensors: fundamental limits, minimal descriptions, and algorithms," in SenSys '06: Proceedings of the 4th international conference on Embedded networked sensor systems, New York, NY, USA, 2006, pp. 251-264, ACM Press.

[4] Wooyoung Kim, Kirill Mechitov, Jeung-Yoon Choi, and Soo Ham, "On target tracking with binary proximity sensors," in IPSN '05: Proceedings of the 4th international symposium on Information processing in sensor networks, Piscataway, NJ, USA, 2005, p. 40, IEEE Press.

[5] Javed Aslam, Zack Butler, Florin Constantin, Valentino Crespi, George Cybenko, and Daniela Rus, "Tracking a moving object with a binary sensor network," in SenSys '03: Proceedings of the 1st international conference on Embedded networked sensor systems, New York, NY, USA, 2003, pp. 150-161, ACM Press.
[6] Wendi Heinzelman, Anantha Chandrakasan, and Hari Balakrishnan, "An application-specific protocol architecture for," IEEE Transactions on Wireless Communications, vol. 1, no. 4, 2002.

[7] Yu-Chee Tseng, Sheng-Po Kuo, Hung-Wei Lee, and Chi-Fu Huang, "Location tracking in a wireless sensor network by mobile agents and its data fusion strategies," The Computer Journal, vol. 47, pp. 448-460, 2004.

[8] Wei-Peng Chen, Jennifer C. Hou, and Lui Sha, "Dynamic clustering for acoustic target tracking in wireless sensor networks.," IEEE Trans. Mob. Comput., vol. 3, no. 3, pp. 258-271, 2004.

[9] Xiaohong Sheng, Yu-Hen Hu, and Parameswaran Ramanathan, "Distributed particle filter with gmm approximation for multiple targets localization and tracking in wireless sensor network," in IPSN '05: Proceedings of the 4th international symposium on Information processing in sensor networks, Piscataway, NJ, USA, 2005, p. 24, IEEE Press.

[10] Petar Djuric, Mahesh Vemula, and Mónica Bugallo, "Tracking with particle filtering in tertiary wireless sensor networks," in ICASSP, Philadelphia, PA, USA, March 18-23 2005.

[11] J. Vermaak, N.D. Lawrence, and P Perez, "Variational inference for visual tracking," in Conf. Computer Vision and Pattern Recog, CVPR'03, June 2003.

[12] Santosh Kumar, Ten H. Lai, and József Balogh, "On kcoverage in a mostly sleeping sensor network," in MobiCom '04: Proceedings of the 10th annual international conference on Mobile computing and networking, New York, NY, USA, 2004, pp. 144-158, ACM Press.

[13] Guanghui He and Jennifer C. Hou, "Tracking targets with quality in wireless sensor networks," in ICNP 2005, 2005.

[14] A. Ihler, J.W. Fisher III, and A.S. Willsky, "Particle filtering under communications constraints," in Proc. Statistical Signal Processing (SSP) 2005, 2005.

[15] Amit S. Chhetri, Darryl Morrell, and Antonia Papandreou Suppappola, "Energy efficient target tracking in a sensor network using non-myopic sensor scheduling," in 7th International Conference on Information Fusion, 2005.

[16] Huaming Wu and Alhussein A. Abouzeid, "Error robust image transport in wireless sensor networks," in 5th Workshop on Applications and Services in Wireless Networks (ASWN 2005), 2005. 